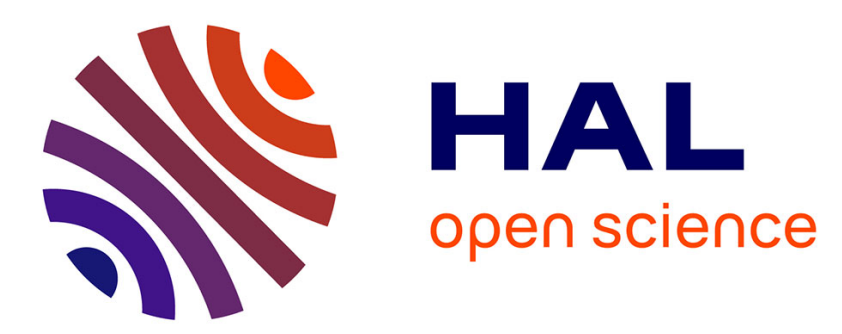

\title{
Étude coût-efficacité du pamoate d'olanzapine: analyse en miroir sur un an
}

\author{
Justine Potaufeu, Bastien Langrée, Dominique Drapier, Gwenola Burgot, \\ Nicolas Marie
}

\section{- To cite this version:}

Justine Potaufeu, Bastien Langrée, Dominique Drapier, Gwenola Burgot, Nicolas Marie. Étude coûtefficacité du pamoate d'olanzapine : analyse en miroir sur un an. L'Encéphale, 2019, 45 (3), pp.232-238. 10.1016/j.encep.2018.11.003 . hal-02020867

\section{HAL Id: hal-02020867 https://hal-univ-rennes1.archives-ouvertes.fr/hal-02020867}

Submitted on 14 Jun 2019

HAL is a multi-disciplinary open access archive for the deposit and dissemination of scientific research documents, whether they are published or not. The documents may come from teaching and research institutions in France or abroad, or from public or private research centers.
L'archive ouverte pluridisciplinaire HAL, est destinée au dépôt et à la diffusion de documents scientifiques de niveau recherche, publiés ou non, émanant des établissements d'enseignement et de recherche français ou étrangers, des laboratoires publics ou privés. 
ETUDE COUT-EFFICACITE DU PAMOATE D'OLANZAPINE : ANALYSE EN MIROIR SUR UN AN COST-EFFECTIVENESS STUDY OF OLANZAPINE PAMOATE: MIRROR-IMAGE ANALYSIS ON ONE YEAR Justine Potaufeu ${ }^{1}$, PharmD, just.potaufeu@gmail.com, j.potaufeu@ch-cotentin.fr Bastien Langrée ${ }^{2}$, PharmD-MSc, b.langree@ch-guillaumeregnier.fr Dominique Drapier ${ }^{3}$, MD-PhD-HDR, d.drapier@ch-guillaumeregnier.fr Gwenola Burgot ${ }^{2}$, PharmD-PhD-Prof, g.burgot@ch-guillaumeregnier.fr Nicolas Marie ${ }^{2}$, PharmD, n.marie@ch-guillaumeregnier.fr

\footnotetext{
${ }^{1}$ Service de Pharmacie, Centre Hospitalier Public du Cotentin - BP 208 - 50102 Cherbourg-en-Cotentin, Tél : 02.33.20.75.49, Fax : 02.33.20.76.84

${ }^{2}$ Service de Pharmacie, Centre hospitalier Guillaume Régnier 108 avenue du général Leclerc 35703 Rennes, Tél : 02.99.33.39.48, Fax : 02.99.33.64.11

${ }^{3}$ Pôle Hospitalo-Universitaire de Psychiatrie Adulte, Centre hospitalier Guillaume Régnier 108 avenue du général Leclerc 35703 Rennes, EA 4712 "comportements et noyaux gris centraux", Université de Rennes 1, Tél : 02.99.33.39.37, Fax : 02.99.33.39.72
} 


\section{Résumé}

Objectif : Les AntiPsychotiques d'Action Prolongée (APAP) ont été développés comme une stratégie pour améliorer l'adhésion thérapeutique des patients, notamment dans la prise en charge de la schizophrénie. Le pamoate d'olanzapine est le seul APAP à avoir un statut de réserve hospitalière en raison du risque de syndrome post-injection. L'enjeu aujourd'hui est la recherche de l'efficience de ces produits de santé, notamment à l'aide de la pharmaco-économie. Cette étude a pour objectif d'évaluer le rapport coût-efficacité du pamoate d'olanzapine dans les conditions réelles de prise en charge.

Méthode : étude coût-efficacité observationnelle rétrospective en miroir sur un an d'une cohorte de 52 patients atteints de schizophrénie et ayant été traités au minimum par trois mois de pamoate d'olanzapine au Centre Hospitalier Guillaume Régnier de Rennes. Le critère principal d'efficacité est le différentiel du nombre d'hospitalisations temps complet avant/après instauration du pamoate d'olanzapine par rapport au différentiel de coûts hospitaliers. Les critères secondaires sont le différentiel du nombre de jours d'hospitalisations et du nombre d'actes ambulatoires entre la période un an avant et un an après.

Résultats: Le pamoate d'olanzapine réduit significativement le nombre d'hospitalisations par rapport à la stratégie de prise en charge antérieure ( $p<0.001$ chez les patients traités plus d'un an). Cette efficacité a un coût supplémentaire non-significatif de 3361 euros par an, en partie dû à son statut de réserve hospitalière.

Conclusion : La stratégie thérapeutique du pamoate d'olanzapine est plus efficace sans majorer le coût de façon significative par rapport à la stratégie thérapeutique antérieure (dont le traitement par olanzapine voie orale).

\section{Abstract}

Introduction: Olanzapine pamoate has a higher cost of treatment than the oral form and requires administration in a hospital setting (unlike others long-acting antipsychotics), and the costeffectiveness of this treatment may be questioned. Many scientific societies and national health systems are increasingly interested in the pharmacoeconomic impact of health products. The search for efficacy of a treatment can be done in two ways: medico-economic modeling studies or observational studies, i.e randomized controlled trials or mirror studies. The models are based on theoretical models from published clinical data, simulating the course and evolution of patient health conditions benefit from a particular therapeutic strategy. Even if the design of observational mirror studies makes it possible to get closer to the clinical reality by observing the patient before and after the initiation of the treatment, the majority of the pharmacoeconomic studies published on olanzapine pamoate are modeling works that do not reflect actual conditions of care. The Guillaume Régnier Hospital Center in Rennes has a large cohort of patients treated with olanzapine pamoate: 121 instauration treatments are recorded from April 1, 2010 to Mars 1, 2015. The objective of this study is to evaluate the cost-effectiveness of olanzapine pamoate in actual clinical practice.

Methods: This is a one year cost-effectiveness retrospective observational mirror-image study of a cohort of 52 patients with schizophrenia who were treated for at least three months with olanzapine pamoate. The primary efficacy endpoint is the differential in the number of full-time hospitalizations before and after the introduction of olanzapine pamoate versus the hospital cost differential. The secondary criteria are the difference of the number of the days spent in hospital and the number of outpatient consultations between the year preceding the injection and the year following it. The 
results were calculated on the general cohort and within 2 subgroups: patients treated for more than one year and those receiving less than one year of treatment with olanzapine pamoate.

Results: Fifty two patients were included (median age $=35$ years, sex ratio $\mathrm{H} / \mathrm{F}=2.7$ ) and only $38.5 \%$ discontinued treatment. For patients who maintained long-acting treatment, they received a dosage of $25 \mathrm{mg}$ oral olanzapine ( $\mathrm{Min}=7.5 \mathrm{mg}$, $\mathrm{Max}=60 \mathrm{mg}$ ), $5 \mathrm{mg}$ more medially than the group having stopped the olanzapine pamoate (20mg; Min $=10 \mathrm{mg}$, Max $=40 \mathrm{mg})$. The majority of these patients were receiving off-label authorized marketing doses of oral olanzapine, and $22 \%$ of them had off-label dosages of olanzapine pamoate. The main causes of discontinuation were symptom persistence, loss of vision and the occurrence of adverse effects (including weight gain and sedation). Olanzapine pamoate significantly reduced the number of hospitalizations compared to the previous management strategy ( 1 less hospitalization, $p<0.001$ in patients treated more than one year and in the general cohort). As a logical consequence the number of hospitalization days in day care increased after the establishment of this long-acting antipsychotic with hospital reserve status (18 in median; Min = 0, Max = 159). We observe a non-statistically significant tendency of decrease in the number of days of full-time hospitalization and an increase in the number of ambulatory procedures, particularly in patients who have maintained the treatment for one year. This efficiency has a nonsignificant additional cost of $€ 3,361$ per year. There is an average multiplication by 8,5 of the drug cost a year later in the general cohort (5.5 in the group of patients treated less than one year and 10.4 in the group of patients who maintained it a year). There is a $23,2 \%$ average increase in the cost of hospitalization in the general cohort $(3.75 \%$ in patients who maintained treatment compared to $48.9 \%$ in patients who discontinued treatment).

Conclusion: By its mirror design, the study is placed in real conditions of care of the patient with schizophrenia. $61.5 \%$ of patients maintained treatment with olanzapine pamoate for a minimum of one year. This APAP is more effective without significantly increasing the cost compared to the previous therapeutic strategy (including oral olanzapine). The additional cost is partly due to the administration restriction in hospital setting in relation to risk of Post-Injection Delirium/Sedation Syndrom (PDSS). There is currently no acceptable efficiency limit. The results of this costeffectiveness analysis can not be extrapolated to the other long-acting antipsychotics since it is the only one with hospital reserve status. The current limitations of medico-economics in psychiatry derive from the heterogeneity of clinical forms and the management of mental pathologies.

Mots clés : pamoate d’olanzapine ; pharmaco-économie ; coût-efficacité ; schizophrénie. Keywords : olanzapine pamoate ; pharmacoeconomics ; cost-effectiveness ; schizophrenia. 


\section{Introduction}

L'adhésion du patient au traitement pharmacologique, matérialisée par l'observance médicamenteuse, est un enjeu majeur dans la prise en charge de la schizophrénie. En 1997, Weiden et al. montrent que le risque de non-observance (partielle ou totale) passe de $50 \%$ à 1 an à $75 \%$ à 2 ans suite à une première hospitalisation [1]. Dans son étude, Lindström et al. constatent que les patients ne prennent que $58 \%$ des doses d'antipsychotiques prescrites [2]. Dans l'étude observationnelle CATIE (Clinical Antipsychotic Trials of Intervention Effectiveness), 74\% des patients atteints de schizophrénie ont arrêté leur traitement après 18 mois, dont $30 \%$ selon leur propre volonté [3]. Ce défaut d'observance est associé à un risque important de rechute; à hauteur de $40 \%$ selon Weiden et al. ou multiplié par 5 sur 2 ans pour Robinson et al. [4-6]. Ainsi, les démarches d'adaptation du traitement pharmacologique (forme galénique, profil d'efficacité et de tolérance) et d'éducation thérapeutique sont essentielles à la prise en charge du patient atteint de schizophrénie, et ceux au même titre que les interventions psychothérapeutiques visant à améliorer les habilités psycho-sociales. Les AntiPsychotiques d'Action Prolongée (APAP) ont été développés pour intégrer une stratégie visant à améliorer l'adhésion thérapeutique des patients. En décembre 2013, un comité d'experts intitulé l'Association Française de Psychiatrie Biologique et de Neuropsychopharmacologie (AFBPN) les a positionnés en première intention dans la stratégie thérapeutique des premières épisodes psychotiques [7]. Cependant, en pratique clinique, ces formes galéniques à libération prolongée restent encore très peu prescrites et notamment chez les patients présentant leur premier épisode psychotique [8-9]. Commercialisé en France en 2010, le pamoate d'olanzapine a obtenu son AMM au regard des résultats de trois études pivots menées par le laboratoire pharmaceutique dont une testant la non-infériorité de cet APAP versus la forme orale [10-12]. Le pamoate d'olanzapine a présenté le même profil de tolérance que la forme orale, auquel vient s'ajouter un risque de syndrome post-injection (Post-Injection Delirium/Sedation Syndrom - PDSS) qui restreint son utilisation au milieu hospitalier.

En France, depuis 2012, la Commission de l'Evaluation Economique et de Santé Publique (CEESP, créée en 2008 au sein de I'HAS) doit émettre un avis d'efficience pour les produits de santé qui ont un ASMR compris entre I et III et qui rapportent plus de 20000 euros de bénéfices au niveau du laboratoire pharmaceutique [13]. La recherche de l'efficience d'un traitement peut se faire selon deux approches: les études de modélisation médico-économique ou les études observationnelles (essais contrôlés randomisés ou études en miroir). Les modélisations se basent sur des modèles théoriques issus de données cliniques publiées, simulant les parcours et l'évolution des états de santé de patients bénéficiant de telle ou telle stratégie thérapeutique. Tandis que les études observationnelles permettent de se rapprocher au mieux de la réalité clinique, notamment avec un design " en miroir " où on observe le patient avant et après l'instauration du traitement à l'étude. Ainsi, avec ce type d'étude en miroir, le biais relatif à l'adhésion thérapeutique du patient est évité [14-15]. Actuellement, les études médico-économiques européennes publiées sur les APAP sont des travaux de modélisation basés sur des simulations de parcours de soins. Malgré des paramètres d'analyse hétérogènes, les modélisations mettent le plus souvent en avant un intérêt pharmacoéconomique notable de ces formes galéniques, en permettant une réduction du nombre de rechutes et donc du coût de prise en charge hospitalier. La rispéridone injectable, qui est l'APAP le plus étudié jusqu'ici, a ainsi un meilleur coût-efficacité par rapport à l'olanzapine orale [16-18]. Peu d'études concernent le pamoate d'olanzapine et encore moins en situation clinique réelle. La particularité de cet APAP est d'être classée en réserve hospitalière, impliquant une hospitalisation de jour et une 
surveillance de 3 heures post-injection, tout en ayant un coût mensuel plus élevé que la forme orale.

\section{Méthodes}

Une étude coût-efficacité observationnelle rétrospective en miroir sur 1 an a été réalisée sur une cohorte de patients présentant un diagnostic de schizophrénie selon la classification CIM-10 et ayant été traités au minimum trois mois par pamoate d'olanzapine au Centre Hospitalier Guillaume Régnier de Rennes (Etablissement Public de Santé Mentale). La période d'étude s'étend du $1^{\mathrm{er}}$ avril 2009 au $1^{\mathrm{er}}$ mars 2015 inclus (avec une première injection postérieure au 1/04/2010 et antérieure au $1 / 03 / 2014)$. Le point de repère du design en miroir correspond à la date de la première injection. On considère que les patients ont maintenu leur traitement durant un an lorsqu'ils ont reçu des injections régulières ( 7 jours de décalage autorisés entre les injections) et qu'ils ont eu moins de trois mois d'arrêt de traitement ; c'est le cas pour 32 d'entre eux (durée médiane de traitement 365 jours). Les données d'activité (hospitalière et ambulatoire) et de coûts hospitaliers sont analysées par comparaison de la période 1 an avant et 1 an après la date repère. Cette analyse médicoéconomique adopte une perspective hospitalière. Le critère principal d'efficacité est le nombre $d^{\prime}$ hospitalisations temps complet avant/après instauration du pamoate d'olanzapine par rapport au différentiel de coûts hospitaliers. Les critères secondaires sont le différentiel du nombre de jours $d^{\prime}$ hospitalisations (temps complet et hospitalisations de jour) et du nombre d'actes ambulatoires entre la période un an avant et un an après.

Les caractéristiques cliniques et démographiques suivantes des patients ont été répertoriées : sexe, âge, l'année de premier contact avec la psychiatrie, le diagnostic psychiatrique et la ville de résidence à l'instauration du pamoate d'olanzapine. Concernant les données relatives à la mise sous traitement APAP, les éléments suivants ont également été recueillis : les posologies d'olanzapine orale à la date précédente la date de la première injection, le nombre d'injections et leurs dosages, les effets indésirables en lien avec l'APAP et, pour les patients ayant arrêté le traitement, les causes d'arrêt ainsi que les alternatives thérapeutiques proposées. Les actes hospitaliers ont été collectés par le Département d'Information Médicale (DIM) de l'établissement. Le recueil a été effectué de la façon suivante :

- Hospitalisation temps Complet (HC) : nombre de jours de présence dans l'unité ainsi que le dernier jour avant la sortie ou un changement de prise en charge du patient.

- Actes à temps partiel : il s'agit des hospitalisations de Jour (HJ) dans notre étude.

- Actes ambulatoires: tous les actes quel que soit l'intervenant et quel que soit le lieu (consultations, démarches administratives, accompagnements de groupe, etc.) hormis les actes réalisés durant l'hospitalisation du patient.

Concernant les hospitalisations temps complet, le choix a été fait de ne pas comptabiliser I'hospitalisation en cours au trois dates clé de la période : $\mathrm{t}-1$ an, to (date de la première injection) et $\mathrm{t}+1$ an . Le coût global médicament a été calculé à partir du prix d'achat hors taxe des médicaments par l'établissement (tarifs 2014 : Zypadhera ${ }^{\circledR} 210 \mathrm{mg}$ : 141,498€, Zypadhera ${ }^{\circledR} 300 \mathrm{mg}: 230,76 €$ et Zypadhera ${ }^{\circledR} 405 \mathrm{mg}$ : $\left.282,996 €\right)$. Le coût global hospitalier a lui été basé sur le coût journalier remboursé par la Sécurité Sociale au $1^{\text {er }}$ août 2014 (355,51€ pour l'HC avec un forfait journalier de $13,50 €$ et $221,25 €$ pour l'HJ). Une analyse statistique à l'aide d'un test de rang des rangs signés de Wilcoxon (formulation bilatérale) a été réalisée avec le logiciel SAS ${ }^{\circledR}$ version 9.4 . 


\section{Résultats}

\section{Caractéristiques des patients}

Cent vingt et un patients ont été traités par pamoate d'olanzapine du $1^{\mathrm{er}}$ avril 2010 au $1^{\mathrm{er}}$ mars 2015 et 52 ont été inclus dans l'étude (cf. figure 1). Au sein de la cohorte, $82 \%$ des patients résidaient en milieu urbain. Selon la classification CIM-10, les quatre formes majoritaires de schizophrénie étaient : la forme paranoïde (15/52), la forme sans précision (10/52), la forme indifférenciée (7/52) et la forme résiduelle (7/52). La posologie d'olanzapine orale avant instauration était de $20 \mathrm{mg}$ en médiane, en sachant que 22 des patients ont reçu des posologies hors-AMM de 25 à $60 \mathrm{mg}$ (cf. tableau I). Sur 936 injections réalisées, plus de la moitié des posologies était de $300 \mathrm{mg}$ tous les 14 jours. Trente-deux patients ont été traités sur un an. Avant instauration du traitement à l'étude, ils recevaient en médiane $5 \mathrm{mg}$ de plus d'olanzapine orale que la cohorte générale. Leur première hospitalisation date en majorité de 2009 et on compte une seule HC avant la date de la première injection. Au sein de ce groupe, 18 patients présentaient des posologies orales antérieures horsAMM et 9 ont reçu des posologies hors-AMM d'APAP ( $405 \mathrm{mg}$ toutes les 2 à 3 semaines). Dans le groupe de patients qui ont arrêté I'APAP précocement, on compte 20 patients avec une durée médiane de traitement de 163 jours ( $M i n=90$ jours, Max $=323$ jours). Les trois causes principales d'arrêt de traitement étaient la persistance des symptômes, la perte de vue des patients et la survenue d'effets indésirables. La quasi-totalité des patients de ce groupe avait été hospitalisée en 2008 pour la première fois (19/20 patients) et présentait deux hospitalisations en médiane sur l'année précédente. Au sein de ce groupe, on retrouve très peu de posologies hors-AMM d'olanzapine orale (4/20 patients) et d'APAP (2/20 patients). Dans chacun des deux groupes, les effets indésirables les plus rapportés au niveau des dossiers patients étaient la prise de poids ( $25 \%$ des patients de chaque groupe) et la sédation (cf. tableau II). Par ailleurs, aucun cas de PDSS n'a été décrit selon la définition du laboratoire.

\section{Activités hospitalières}

En analysant les données d'activité hospitalière sur les deux périodes de l'étude en miroir, on constate une diminution significative du nombre d'hospitalisation temps complet; soit une hospitalisation $\mathrm{HC}$ en moins en médiane dans la cohorte (cf. tableau III et IV). Le nombre d'admissions en hospitalisation HC est plus important dans le sous-groupe qui a arrêté l'olanzapine d'action prolongée en cours d'année que dans le sous-groupe qui l'a maintenu au minimum un an. Concernant le nombre de jours d'hospitalisation, les résultats sont plus hétérogènes et nonsignificatifs. On observe néanmoins une réduction significative de 33.5 jours d'HC chez 22 patients qui ont maintenu le traitement durant un an $(p<0.001)$. Tandis que pour le groupe avec arrêt de traitement, on constate une augmentation de 11 jours d'HC en médiane sur le différentiel un avant/un an après. Dans les deux sous-groupes, des durées maximales de 354 à 363 jours d'HC montrent un maintien de l'hospitalisation chez certains patients qu'ils soient sous pamoate d'olanzapine ou non. Le pamoate d'olanzapine étant administré en hôpital de jour, on assiste à une augmentation significative du nombre d'HJ après son instauration au sein des trois groupes de patients. De façon logique, on constate que l'augmentation la plus importante concerne les patients qui ont maintenu le traitement durant un an. La différence de nombre d'actes ambulatoires n'est pas significative entre les deux périodes, même si elle a tendance à être augmentée après instauration du pamoate d'olanzapine. 


\section{Données économiques}

La dispersion des valeurs étant importante (médianes très différentes des moyennes, tableau V), nous utiliserons les moyennes des coûts par patient comme valeurs indicatives pour décrire une tendance à l'augmentation des coûts un an après. En moyenne, le coût médicamenteux a été multiplié par 8,5 un an après dans la cohorte générale (par 5,5 dans le groupe des patients qui ont arrêté le traitement et par 10,4 dans le groupe des patients qui l'ont maintenu minimum un an). Le coût attribué à l'hospitalisation est en moyenne augmenté de $23,2 \%$ dans la cohorte générale (3,75\% chez les patients qui ont maintenu le traitement contre $48,9 \%$ chez les patients qui l'ont arrêté). Cette tendance à l'augmentation des coûts n'est pas toujours confirmée par le test de Wilcoxon basé sur la comparaison des médianes des différences de coût total avant/après instauration de I'APAP. Ainsi, dans chacun des groupes de l'étude, le coût global hospitalier est majoré un an après et la différence de coût se situe en médiane à 3361 euros dans la cohorte générale $(p=0,082), 2186$ euros dans le groupe ayant maintenu le pamoate d'olanzapine $(p=0,57)$ et 15337 euros dans le groupe qui l'a arrêté $(p<0,05)$. En se focalisant sur les 22 patients qui ont maintenu le traitement et qui n'ont pas été ré-hospitalisés dans l'année qui suivait l'instauration, on constate une réduction non-significative de 4355 euros $(p=0,129)$.

\section{Discussion}

L'étude observationnelle rétrospective réalisée sur une cohorte de 52 patients montre une efficacité de l'olanzapine d'action prolongée sur le critère principal de l'étude, c'est-à-dire le nombre d'hospitalisations temps complet. De la même façon, la majorité des études d'efficacité réalisées sur les APAP avec un design en miroir (notamment la rispéridone, la palipéridone et l'aripiprazole à action prolongée) sont en accord avec cette réduction globale du nombre de réhospitalisation après instauration [15, 19-24]. Concernant les critères secondaires, on observe une augmentation significative des hospitalisations de jour en lien avec l'administration du pamoate d'olanzapine. Les différences non-significatives ne nous permettent pas de conclure mais montre une tendance à la diminution de la durée de séjour et à l'augmentation de l'activité ambulatoire notamment chez les patients qui ont maintenu le traitement durant un an. On note un taux important de maintien du traitement pendant minimum un an : $61,5 \%$ de la cohorte générale. Les effets indésirables recensés au sein de la cohorte sont ceux qui sont le plus décrits avec l'olanzapine et on n'a pas observé de relation dose-effet (notamment avec les posologies hors-AMM). Pour certains patients, la mise sous APAP n'a pas permis de les désinstitutionnaliser. Contrairement aux patients qui ont été traités minimum un an, l'histoire de la maladie des patients qui ont arrêté le traitement semblaient plus ancienne et plus sévère. De plus, la posologie antérieure d'olanzapine orale dans ce groupe était inférieure à la posologie orale du groupe ayant maintenu le pamoate d'olanzapine $(20 \mathrm{mg} / \mathrm{j}$ versus $25 \mathrm{mg} / \mathrm{j}$ en hors-AMM). On peut donc se demander si les patients qui ont arrêté le traitement ne présentaient pas une forme résistante de la pathologie, n'étaient pas suffisamment équilibrés sous olanzapine ou n'étaient pas suffisamment adhérents au traitement par pamoate d'olanzapine et à ses modalités d'injection. De nombreuses études corroborent ces deux premières hypothèses. En effet, il a été démontré que l'olanzapine orale était utilisée à des doses élevées hors AMM dans des cas de résistance, notamment en milieu hospitalier et entrainait des réponses cliniques satisfaisantes, sans compromettre la bonne tolérance neurologique au traitement [25-27]. Enfin, ces résultats renforcent l'idée que la prise en charge de ces patients ne doit pas reposer uniquement sur 
le traitement pharmacologique mais également sur des démarches éducatives, psycho-sociales de réhabilitation et de réinsertion sociale.

La médiane du coût annuel hospitalier des patients traités par pamoate d'olanzapine est de 27621 euros (Min = 3 312, Max = 136857 euros). Elle se rapproche de celle calculée pour les patients hospitalisés dans l'étude de Raymond et al. de 2009 [28] : 51356 euros en coûts médicaux et transports (coûts de transport non inclus dans notre étude). On voit nettement que la part médicamenteuse est plus importante avec l'utilisation des APAP en termes de dépenses de santé, en effet elle passe de $1,7 \%$ à $15,1 \%$ du coût global hospitalier sur la cohorte générale en médiane $(1,6 \%$ à $10 \%$ du coût global en moyenne sur la cohorte générale et $1,7 \%$ à $14,9 \%$ en moyenne dans le groupe des patients ayant maintenu le traitement un an). La durée d'hospitalisation temps complet semble diminuer alors que le coût hospitalier global augmente; cela s'explique par une augmentation de l'activité d'hospitalisation de jour due au risque de PDSS, ainsi que par l'augmentation du coût médicamenteux. En effet, le pamoate d'olanzapine est le seul APAP qui présente un risque de syndrome post-injection imposant une surveillance hospitalière de 3 heures minimum (détection des signes et symptômes de surdosage). Son efficacité (réduction d'une hospitalisation sur l'année) a un coût supplémentaire annuel non-significatif pour l'assurance maladie de 3361 euros en médiane, soit 15,6\% du coût annuel hospitalier (21 505 euros, avant l'instauration du pamoate d'olanzapine). Chez les patients qui ont maintenu le traitement durant un an, on observe également une augmentation non-significative du coût de 2186 euros. La seule réduction de coût est réalisée dans le cas de patients ayant maintenu le traitement un an sans avoir été admis en hospitalisation temps complet, mais elle demeure non-significative. D'après la représentation graphique de I'ICER proposée par Woronoff-Lemsi et al. [29], la stratégie thérapeutique du pamoate d'olanzapine est plus efficace et plus coûteuse que la stratégie thérapeutique antérieure (dont le traitement par olanzapine voie orale) mais reste une alternative acceptable. Actuellement, les études internationales de coût-efficacité avec une méthodologie en miroir concernent essentiellement la rispéridone d'action prolongée et présentent des résultats mitigés [19-22, 30]. II est cependant difficile de comparer nos résultats à ces études car elles utilisent des méthodologies variables (inclusion des coûts indirects de la pathologie ou non, critères d'efficacité cliniques ou non, prise en compte du taux d'abandon ou non, etc.) et concernent des systèmes de santé différents.

L'étude Coût-Efficacité du pamoate d'olanzapine est réalisée en "miroir » où le patient est son propre contrôle. En effet, le concept d'étude en miroir permet de se situer dans les conditions réelles de prise en charge et d'adhésion au traitement, notamment en psychiatrie (effectiveness). Concernant les limites de l'étude, sa durée en est une, puisqu'elle est de deux ans alors qu'il s'agit d'une affection chronique. L'étude est seulement monocentrique. Il existe un biais de recrutement, puisque la majorité des instaurations de traitement ont été réalisées chez des patients vivant en milieu urbain (injection intra-hospitalière contraignante pour les patients habitants à distance des unités d'hospitalisation). Nous avons choisi comme élément discriminant le nombre d'admission en hospitalisation temps complet car c'est un critère accessible à l'analyse, qui peut refléter des rechutes de la pathologie psychiatrique et un échec de la prise en charge thérapeutique antérieure. Les données cliniques utilisées (tolérance et causes d'arrêt du traitement) ne sont pas exhaustives puisqu'elles reposent exclusivement sur les observations médicales renseignées dans le dossier médical informatique. Ainsi, on peut penser que la nature et la fréquence des effets indésirables sont sous-estimés, notamment en terme de perturbations biologiques $(25 \%$ de prise de poids et 
seulement trois perturbations du bilan lipidique/glucidique dans la cohorte générale, cf. tableau II). L'étude présente également un biais de sélection puisque le pamoate d'olanzapine a été instauré suite à une inefficacité des thérapeutiques antérieures (dont l'olanzapine orale) et nous n'avions aucun contrôle sur les médicaments pris auparavant. De plus, on sait que dans la prise en charge de la schizophrénie, les co-prescriptions sont nombreuses et peuvent influencer sur l'efficacité et la tolérance du traitement, nous ne les avons pas répertoriés ici. Le statut de réserve hospitalière induit également un biais de mode de prise en charge puisqu'il fait forcément appel à la mobilisation de ressources hospitalières. Nous avons fait le choix de ne pas inclure les coûts ambulatoires car nous nous sommes heurtés à des problèmes de faisabilité (non-accès aux données de la caisse d'Assurance Maladie) et parce qu'ils semblent être bien inférieurs aux coûts directs hospitaliers [28]. Cependant, il manque l'intégration des coûts indirects (prestations sociales, indemnités d'arrêt de travail, etc.) au sein de notre analyse. Un autre point limitant concerne les coûts directs hospitaliers qui se résument ici aux coûts des traitements médicamenteux ajoutés à celui du tarif hospitalier de la sécurité sociale. On a bien conscience qu'il ne s'agit pas des coûts hospitaliers réels, cependant, il est difficile de s'approcher de la réalité avec le système de la DAF (Dotation Annuelle de Financement) qui finance actuellement la psychiatrie en France.

\section{Conclusion}

Par son design en miroir, l'étude se place dans des conditions réelles de prise en charge du patient atteint de schizophrénie. $61,5 \%$ des patients ont maintenu le traitement par pamoate d'olanzapine pendant minimum un an. L'analyse médico-économique montre que cet APAP réduit significativement le nombre d'hospitalisations temps complet par rapport à la stratégie thérapeutique antérieure (dont le traitement par olanzapine voie orale) avec un ICER calculé à + 3361 euros par an en médiane. Cette différence de coût direct est non significative et s'explique en partie par la restriction d'administration de I'APAP en milieu hospitalier. Les résultats de cette analyse coût-efficacité ne sont pas extrapolables aux autres APAP puisqu'il est le seul à détenir un statut de réserve hospitalière. II n'existe pas actuellement de limite d'efficience acceptable. Les limites actuelles de la médico-économie en psychiatrie proviennent de l'hétérogénéité des formes cliniques et des prises en charge des pathologies mentales. 


\section{TABLE DES FIGURES}

Figure 1: Processus de sélection des sujets de l'étude coût-efficacité en miroir du pamoate d'olanzapine sur un an. 


\section{Bibliographie:}

[1] Weiden P, Glazer W. Assessment and treatment selection for " revolving door " inpatients with schizophrenia. Psychiatr Q 1997; 68 (4): 377-92.

[2] Lindstrom E, Bingefors K. Patient compliance with drug therapy in schizophrenia: economic and clinical issues. Pharmacoeconomics 2000; 18: 105-24.

[3] Lieberman JA, Stroup TS, Mcevoy JP, et al. Effectiveness of antipsychotic drugs in patients with chronic schizophrenia. N Engl J Med 2005; 353 (12): 1209-23.

[4] Weiden P, Olfson M. Cost of relapse in schizophrenia. Schizophrenia Bulletin 1995; 21: 419-29.

[5] Robinson D, Woerner MG, Alvir JM, et al. Predictors of relapse following response from a first episode of schizophrenia or schizoaffective disorder. Arch Gen Psychiatry 1999; 56 (3): 241-47.

[6] Misdrahi D. L’observance thérapeutique : un objectif essentiel. L’Encéphale 2006; 32: 1076-9.

[7] Samalin L, Abbar M, Courtet $P$, et al. Recommandations Formalisées d'Experts de l'AFPBN : prescription des neuroleptiques et antipsychotiques d'action prolongée. L'Encéphale 2013; 39 Suppl: 189-203.

[8] Heres S, Lambert M, Vauth R. Treatment of early episode in patients with schizophrenia: the role of long acting antipsychotics. Eur Psychiatry 2014; 29 Suppl: 1409-13.

[9] Stahl SM. Long-acting injectable antipsychotics: shall the last be first? CNS Spectr 2014; 19 (1): 3-5.

|10] Lauriello J, Lambert T, Andersen S, et al. An 8-week, double-blind, randomized, placebocontrolled study of olanzapine long-acting injection in acutely ill patients with schizophrenia. J Clin Psychiatry 2008; 69 (5): 790-9.

[11] Kane JM, Detke HC, Naber D, et al. Olanzapine long-acting injection: a 24-week, randomized, double-blind trial of maintenance treatment in patients with schizophrenia. Am J Psychiatry 2010; 167 (2): 181-9.

[12] McDonnell DP, Landry J, Detke HC. Long-term safety and efficacy of olanzapine long-acting injection in patients with schizophrenia or schizoaffective disorder: a 6-year, multinational, singlearm, open-label study. Int Clin Psychopharmacol 2014; 29 (6): 322-31.

[13] Décret $n^{\circ}$ 2012-1116 du 2 octobre 2012 relatif aux missions médico-économiques de la Haute Autorité de santé. 2012.

[14] Kane JM, Kishimoto T, Correll CU. Assessing the comparative effectiveness of long-acting injectable vs. oral antipsychotic medications in the prevention of relapse provides a case study in comparative effectiveness research in psychiatry. J Clin Epidemiol 2013; 66 (8) Suppl: 37-41.

[15] Kishimoto $T$, Nitta $M$, Borenstein $M$, et al. Long-acting injectable versus oral antipsychotics in schizophrenia: a systematic review and meta-analysis of mirror-image studies. J Clin Psychiatry 2013; 74 (10): 957-65. 
[16] De Graeve D, Smet A, Mehnert A, et al. Long-acting risperidone compared with oral olanzapine and haloperidol depot in schizophrenia: a Belgian cost-effectiveness analysis. PharmacoEconomics 2005; 23 Suppl: 35-47.

[17] Llorca PM, Miadi-Fargier H, Lançon C, et al. Cost-effectiveness analysis of schizophrenic patient care settings: impact of an atypical antipsychotic under long-acting injection formulation. L’Encéphale 2005; 31 (2): 235-46.

[18] Laux G, Heeg B-M, Van Hout B-A, et al. Costs and effects of long-acting risperidone compared with oral atypical and conventional depot formulations in Germany. PharmacoEconomics 2005; 23 Suppl: 49-61.

[19] Olivares JM, Rodriguez-Martinez A, Burón JA, et al. E-STAR Study Group. Cost-effectiveness analysis of switching antipsychotic medication to long-acting injectable risperidone in patients with schizophrenia : a 12- and 24-month follow-up from the e-STAR database in Spain. Appl Health Econ Health Policy 2008; 6 (1): 41-53.

[20] Wu DB-C, Lee EHM, Chung WS, et al. Cost analysis of risperidone long-acting injection in the treatment of schizophrenia and schizoaffective disorders in Hong Kong: An approach using generalised estimating equations. Psychiatry Res 2013; 210 (3): 745-50.

[21] Taylor D, Fischetti C, Sparshatt A, et al. Risperidone long-acting injection: a 6-year mirror-image study of healthcare resource use. Acta Psychiatr Scand 2009; 120 (2): 97-101.

[22] Carswell C, Wheeler DA, Vanderpyl J, et al. Comparative Effectiveness of Long-Acting Risperidone in New Zealand. Clin Drug Investig 2012; 30 (11): 777-87.

[23] Zhang F, Si T, Chiou C-F, et al. Efficacy, safety, and impact on hospitalizations of paliperidone palmitate in recent-onset schizophrenia. Neuropsychiatric Disease and Treatment 2015; 11: 657-68.

[24] Kane JM, Zhao C, Johnson BR, et al. Hospitalization rates in patients switched from oral antipsychotics to aripiprazole once-monthly: final efficacy analysis. J Med Econ 2015; 18: 145-54.

[25] Lerner V. High-dose olanzapine for treatment-refractory schizophrenia. Clin Neuropharmacol 2003; 26 (2): 58-61.

[26] Qadri SF, Padala PR, Strunk JC, et al. High-Dose Olanzapine Orally Disintegrating Tablets for Treatment-Resistant Psychosis. Prim Care Companion J Clin Psychiatry 2006; 8 (4): 244-245.

[27] Batail JM, Bleher S, Lozachmeur C, et al. Very-high-dose olanzapine for treatment-resistant schizophrenia. Open J Psychiatry 2012; 2: 269-71.

[28] Raymond S, Martin M, Netillard C, et al. Schizophrénie: évaluation du coût de la prise en charge. J Econ Medicale 2009; 27 (7-8): 421-33. 
[29] Woronoff-Lemsi M-C, Limat S, Husson M-C. Approche pharmaco-économique : évaluation pharmao-médico-économique de stratégies thérapeutiques : éléments de méthodologie. Doss. CNHIM 2000; 40-51.

[30] Kamat SA, Offord S, Docherty J, et al. Reduction in inpatient resource utilization and costs associated with long-acting injectable antipsychotics across different age groups of Medicaid-insured schizophrenia patients. Drugs in Context 2015; 4: 212-67. 


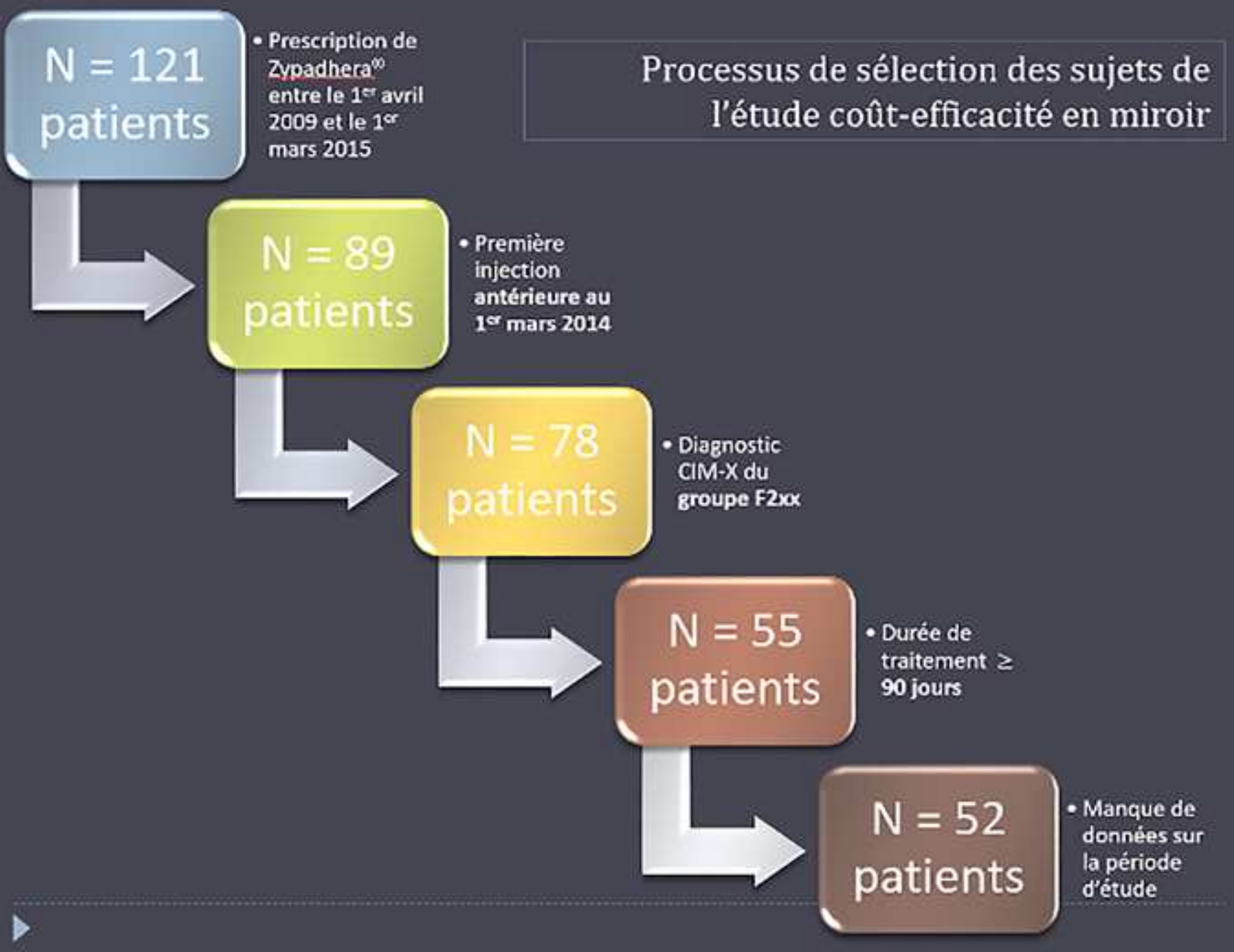


Tableau I : Caractéristiques des patients de l'étude coût-efficacité en miroir du pamoate d'olanzapine sur un an.

\begin{tabular}{|c|c|c|c|}
\hline & $\begin{array}{c}\text { Cohorte générale } \\
n=52\end{array}$ & $\begin{array}{l}\text { Patients traités }>1 \text { an } \\
n=32\end{array}$ & $\begin{array}{c}\text { Patients traités }<1 \text { an } \\
n=20\end{array}$ \\
\hline Sex-ratio H/F & 2,7 & 3 & 2,3 \\
\hline $\begin{array}{l}\text { Age d'instauration du } \\
\text { Zypadhera }^{\circledR} \text { (médiane) }\end{array}$ & 35 ans & 35 ans & 36 ans \\
\hline $\begin{array}{l}\text { Année de premier } \\
\text { contact avec la } \\
\text { psychiatrie (médiane, } \\
\text { nombre de patients } \\
\text { pour lesquels l'année } \\
\text { est connue) }\end{array}$ & $2004(41 / 52)$ & $2009(28 / 32)$ & $2008(19 / 20)$ \\
\hline $\begin{array}{l}\text { Posologie d'olanzapine } \\
\text { orale avant instauration } \\
\text { (médiane, (min-max)) }\end{array}$ & $\begin{array}{c}20 \mathrm{mg} \\
(7,5-60 \mathrm{mg})\end{array}$ & $\begin{array}{c}25 \mathrm{mg} \\
(7,5-60 \mathrm{mg})\end{array}$ & $\begin{array}{c}20 \mathrm{mg} \\
(10-40 \mathrm{mg})\end{array}$ \\
\hline $\begin{array}{l}\text { Nombre de patients } \\
\text { avec des posologies } \\
\text { orales hors AMM }\end{array}$ & 22 & 18 & 4 \\
\hline $\begin{array}{l}\text { Nombre d'injections de } \\
\text { Zypadhera }^{\circledR}\end{array}$ & 936 & 692 & 224 \\
\hline $\begin{array}{l}\text { Nombre de patients } \\
\text { avec des injections de } \\
\text { Zypadhera }{ }^{\circledR} \text { hors AMM }\end{array}$ & $\begin{array}{c}11 \\
\text { (141 injections) }\end{array}$ & $\begin{array}{c}9 \\
\text { (133 injections) }\end{array}$ & $\begin{array}{c}2 \\
\text { (8 injections) }\end{array}$ \\
\hline $\begin{array}{l}\text { Nombre } \\
\text { d'hospitalisations } \\
\text { l'année précédente } \\
\text { (médiane) }\end{array}$ & $N(H C)=1$ & $N(H C)=1$ & $N(H C)=2$ \\
\hline
\end{tabular}


Tableau II : Principaux effets indésirables rencontrées dans l'étude coût-efficacité en miroir du pamoate d'olanzapine sur un an.

\begin{tabular}{|l|c|c|c|}
\hline Effets indésirables & $\begin{array}{c}\text { Cohorte } \\
\text { générale } \\
\mathbf{N}=\mathbf{5 2}\end{array}$ & $\begin{array}{c}\text { Patients } \\
\text { traités } \mathbf{2} \mathbf{1} \text { an } \\
\mathbf{N}=\mathbf{3 2}\end{array}$ & $\begin{array}{c}\text { Patients } \\
\text { traités } \\
\mathbf{N} \mathbf{1} \text { an }\end{array}$ \\
\hline Prise de poids & 13 & 8 & 5 \\
\hline Sédation & 10 & 6 & 4 \\
\hline $\begin{array}{l}\text { Ralentissement psychique/ troubles de la } \\
\text { mémoire }\end{array}$ & 6 & 6 & 0 \\
\hline Troubles digestifs* & 5 & 4 & 1 \\
\hline Insomnie & 4 & 4 & 0 \\
\hline $\begin{array}{l}\text { Troubles du métabolisme } \\
\text { lipidique/glucidique }\end{array}$ & 3 & 3 & 1 \\
\hline Troubles sexuels & 3 & 2 & 1 \\
\hline Réaction à l'injection & 2 & $1^{\text {a }}$ & \\
\hline Malaise/vertiges & 2 & 1 & 1 \\
\hline Mictions fréquentes & 1 & 0 & 1 \\
\hline $\begin{array}{l}\text { TOTAL } \\
\text { *: troubles digestifs récurrents de type } \\
\text { nausées/vomissements et douleurs } \\
\text { abdominales. Les cas de constipations } \\
\text { occasionnelles ne sont pas recensés. } \\
\text { a : Bouffée de chaleur à l'injection, } \\
\text { b : Syndrome d'hyper-sédation post- } \\
\text { injection. }\end{array}$ & 49 & 35 & \\
\hline
\end{tabular}


Tableau III : Critères d'évaluation primaire et secondaire (médiane, valeurs minimale et maximale) avant et après instauration du pamoate d'olanzapine.

\begin{tabular}{|c|c|c|c|c|c|c|c|c|}
\hline & \multicolumn{2}{|c|}{$\begin{array}{l}\text { Nombre d'hospitalisation } \\
\text { temps complet } n(\mathrm{HC})\end{array}$} & \multicolumn{2}{|c|}{$\begin{array}{c}\text { Nombre de jours } \\
\text { d'hospitalisation temps } \\
\text { complet nj }(\mathrm{HC})\end{array}$} & \multicolumn{2}{|c|}{$\begin{array}{l}\text { Nombre d'hospitalisations } \\
\text { de jour } n(H J)\end{array}$} & \multicolumn{2}{|c|}{$\begin{array}{l}\text { Nombre d'actes ambulatoires } \\
\qquad \mathrm{n} \text { (actes) }\end{array}$} \\
\hline & $A n-1$ & $A n+1$ & $A n-1$ & $A n+1$ & An-1 & $A n+1$ & An-1 & $A n+1$ \\
\hline $\begin{array}{l}\text { Cohorte générale } \\
n=52\end{array}$ & $1(0-6)$ & $0(0-3)$ & $55(0-363)$ & $34,5(0-363)$ & $0(0-70)$ & $18(0-159)$ & $12,5(0-164)$ & $13(0$ - 190) \\
\hline $\begin{array}{l}\text { Patients traités } \geq 1 \text { an } \\
n=32\end{array}$ & $1(0-3)$ & $0(0-2)$ & $55(0-360)$ & $14,5(0-354)$ & $0(0-70)$ & $19(0-159)$ & $10(0-164)$ & $14,5(0-190)$ \\
\hline $\begin{array}{l}\text { Patients traités }<1 \text { an } \\
n=20\end{array}$ & $2(0-6)$ & $0,5(0-3)$ & $59(6-363)$ & $90(1-363)$ & $0(0-30)$ & $9(0-67)$ & $14,5(0-57)$ & $12,5(0-64)$ \\
\hline
\end{tabular}


Tableau IV : Comparaison des critères d'évaluation avant et après instauration du pamoate d'olanzapine selon le test de rang de Wilcoxon

\begin{tabular}{lcccc}
\hline & $\mathbf{n}(\mathrm{HC})$ & $\mathrm{nj}(\mathrm{HC})$ & $\mathrm{n}(\mathrm{HJ})$ & $\mathbf{n}($ actes $)$ \\
\hline $\begin{array}{l}\text { Cohorte générale } \\
\mathrm{n}=52\end{array}$ & $\mathbf{P}<\mathbf{0 , 0 0 1}$ & $\mathrm{P}=0,6734$ & $\mathbf{P}<\mathbf{0 , 0 0 1}$ & $\mathrm{P}=0,4175$ \\
$\begin{array}{l}\text { Patients traités } \geq 1 \text { an } \\
\mathrm{n}=32\end{array}$ & $\mathbf{P}<\mathbf{0 , 0 0 1}$ & $\mathrm{P}=0,0942$ & $\mathbf{P}<\mathbf{0 , 0 0 1}$ & $\mathrm{P}=0,3501$ \\
$\begin{array}{l}\text { Patients traités }<1 \text { an } \\
\mathrm{n}=20\end{array}$ & $\mathbf{P}<\mathbf{0 , 0 1}$ & $\mathrm{P}=0,2121$ & $\mathbf{P}<\mathbf{0 , 0 0 1}$ & $\mathrm{P}=0,8808$ \\
\hline
\end{tabular}


Tableau V : Coût direct hospitalier annuel par patient en termes d'usage de médicaments, d'hospitalisations (HC : hospitalisation temps complet, HJ : hospitalisation de jour) et coût total avant et après l'instauration du pamoate d'olanzapine (de haut en bas : moyenne, médiane, valeurs minimale et maximale).

\begin{tabular}{|c|c|c|c|c|c|c|}
\hline \multirow{2}{*}{$\begin{array}{l}\text { Coût } \\
\text { annuel } \\
\text { par } \\
\text { patient }\end{array}$} & \multicolumn{2}{|c|}{$\begin{array}{l}\text { Cohorte générale } \\
\qquad N=52\end{array}$} & \multicolumn{2}{|c|}{$\begin{array}{l}\text { Patients traités } \geq 1 \text { an } \\
\qquad N=32\end{array}$} & \multicolumn{2}{|c|}{$\begin{array}{l}\text { Patients traités }<1 \text { an } \\
\qquad N=20\end{array}$} \\
\hline & $T-1$ an & $T+1$ an & $\mathrm{T}-1$ an & $T+1$ an & $\mathrm{T}-1$ an & $T+1$ an \\
\hline 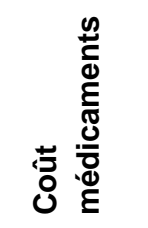 & $\begin{array}{c}504 € \\
356 € \\
(0-3703)\end{array}$ & $\begin{array}{c}4277 € \\
4171 € \\
(852-7845)\end{array}$ & $\begin{array}{c}503 € \\
356 € \\
(0-3703)\end{array}$ & $\begin{array}{c}5209 € \\
5263 € \\
(3208-7845)\end{array}$ & $\begin{array}{c}505 € \\
351 € \\
(24-2351)\end{array}$ & $\begin{array}{c}2787 € \\
3066 € \\
(852-4316)\end{array}$ \\
\hline 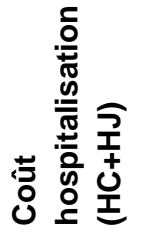 & $\begin{array}{c}31079 € \\
20996 € \\
(0-133951)\end{array}$ & $\begin{array}{c}38283 € \\
23647 € \\
(1697-133951)\end{array}$ & $\begin{array}{c}28747 € \\
20996 € \\
(0-132844)\end{array}$ & $\begin{array}{c}29826 € \\
14610 € \\
(3983-130630)\end{array}$ & $\begin{array}{c}34808 € \\
21772 € \\
(2435-133951)\end{array}$ & $\begin{array}{c}51814 € \\
\mathbf{4 3} \mathbf{4 9 9 €} \\
(1697-133951)\end{array}$ \\
\hline $\begin{array}{l}\bar{\sigma} \\
\text { के } \\
5 \\
0 \\
0\end{array}$ & $\begin{array}{c}31582 € \\
21505 € \\
(0-135072)\end{array}$ & $\begin{array}{c}42560 € \\
27624 € \\
(3312-136857)\end{array}$ & $\begin{array}{c}29250 € \\
21385 € \\
(0-134628)\end{array}$ & $\begin{array}{c}35034 € \\
18530 € \\
(8145-136857)\end{array}$ & $\begin{array}{c}35314 € \\
22287 € \\
(2460-135072)\end{array}$ & $\begin{array}{c}54601 € \\
46910 € \\
(3312-136790)\end{array}$ \\
\hline
\end{tabular}


Formulaire : Conflit $\mathbf{d}$ interet / Conflict of Interest form

Déclaration d'intérêts : les auteurs ont déclaré n'avoir aucun lien d'intérêt en lien avec cet article. 